\title{
UN NUEVO HIBRIDO DE TECTARIA (FILICALES: TECTARIACEAE) EN COSTA RICA
}

\author{
Alexander Fco. Rojas Alvarado \\ Universidad de Costa Rica, Sede de Occidente. Apdo. 111-4250, San Ramón, Alajuela. \\ Dirección actual: Museo Nacional de Costa Rica, Depto. de Historia Natural \\ Apdo. 749-1000, San José, Costa Rica. afrojasa@hotmail.com
}

\begin{abstract}
A new hybrid between Tectaria acutiloba and T. lizarzaburui from the Atlantic lowlands of Costa Rica is described. The concepts of T. lizarzaburui and T. vivipara are discussed, and the use of the former name is proposed to replace T. vivipara in continental neotropics.
\end{abstract}

Resumen. Aquí se describe un nuevo híbrido entre Tectaria acutiloba y T. lizarzaburui, de las llanuras del Caribe de Costa Rica. Se discuten los conceptos de T. lizarzaburui y T. vivipara y se propone el uso del primero de estos nombres en el neotrópico continental.

Key words / Palabras Clave: Pteridophyta, Tectariaceae, Tectaria, helechos híbridos, hybrid ferns, Costa Rica

Existen tres híbridos previamente registrados en Tectaria. El primero es X Pleuroderris michleriana (Wagner et al. 1978), un híbrido entre Dictioxiphium panamense Hook. [= Tectaria panamensis (Hook.) R.M. Tryon \& A.F. Tryon] y Tectaria incisa Cav. Este híbrido es fácil de reconocer dado que $D$. panamense tiene frondas simples y soros lineares en el margen, mientras que $T$. incisa es pinnada con soros redondos y distribuidos en el envés de la lámina. Como resultado, el híbrido tiene la lámina pinnatífida a pinnada con el ápice pinnatífido; los soros comúnmente son oblongos y cercanos al margen. Muchos nombres han sido utilizados para este singular híbrido, como son Aspidium tatei (Baker) Diels, Hypoderris heteroneuroides H. Christ, H. seemannii Prent., Phegopteris tatei (Baker) Salomon, Polypodium tatei Baker y Tectaria tatei (Baker) C. Chr. (Moran 1995, Wagner et al. 1978).

El segundo híbrido fue descrito por Gómez (1977). Supuestamente, tanto Tectaria draconoptera (D.C. Eaton) Copel. como T. nicotianifolia (Baker) C. Chr. hibridizan con T. nicaraguensis (E. Fourn.) C. Chr. para producir el híbrido T. x cynthiae L.D. Gómez, que se caracteriza por el estípite densamente escamoso y la lámina irregular e imbricadamente lobada, además de la presencia de esporas abortadas.
El tercer híbrido fue descrito en 1985 como Tectaria $\times$ bulbifera Jermy \& T.G. Walker $(=T$. incisa $\times$ T. vivipara). Se puede reconocer por tener yemas como en $T$. vivipara, aunque más pequeñas; por el aporte genético de $T$. incisa es más robusta que $T$. vivipara y tiene costa y cóstulas glabras. Agregado a lo anterior, el híbrido tiene esporas abortivas y un número cromosómico de $\mathrm{n}=120$, por lo cual es un triploide.

El nuevo híbrido, descrito a continuación, ha sido considerado como tal después de comparar táxones afines en distintos tratamientos taxonómicos para Mesoamérica y Sudamérica (Smith 1981, Stolze 1981, Mickel \& Beitel 1988, Murillo \& Harker 1990, Moran 1995, Smith 1995, Tryon \& Stolze 1991), en publicaciones recientes que incluyen a la familia Tectariaceae (Grayum 1987, Moran 1990, 1992, Rojas 2001) y después de revisar las colecciones del Herbario Nacional de Costa Rica (CR) y del Instituto Nacional de Biodiversidad (INB).

Tectaria $\times$ chaconiana A. Rojas, nothosp. nova

TIPO: Costa Rica. Heredia: Sarapiquí, Puerto Viejo, Estación Biológica La Selva, cercanías de La Arboleda, orillas de quebrada, $10^{\circ} 26^{\prime} 00^{\prime} \mathrm{N}$, 8401'00"W, 50 m, 2 dic 1997, A. Rojas et al. 4156 (Holotipo: INB, isotipos: CR, MO, NY). FIG. 1, 2. 
Nothospecies nova a T. acutiloba (Hieron.) Maxon rhizomatis squamis minoribus [1.5-3 mm versus (3-) $5-10 \mathrm{~mm}$ ], fuscis obscura (vs. pardalis) et superficies glabrescentis (vs. pubescentis), frondibus longioribus (1-1.4 m vs. 0.5-1 m), pinnis minus divisis (pinnato-lobulatis vs. pinnato-pinnatifida) et prolifera gemmae (vs. absque gemmae) in axillae pinnae basalis divergens.

Rizoma de 7-15 mm de diámetro, erecto; escamas del rizoma 1.5-3 $\mathrm{mm}$ de largo, lanceoladas, pardo oscuro, margen entero; frondas de 1-1.4 m de largo; estípite más corto que la lámina o tan largo como ella, pardo o raramente pajizo, finamente peloso, las escamas de 2-5 $\mathrm{mm}$ de largo, lanceoladas, glabras; lámina de (18-) 25-50 (-60) x 15-30 cm, deltada, bipinnado-pinnatífida, con yemas en el primer par de pinnas; segmento apical cuneado a anchamente redondeado; pares de pinnas 5-7, opuestas o subopuestas, lobadas a pinnado-lobuladas, las superiores decurrentes; pinnas basales 10-40 × 5-32 cm, lanceoladas, pinnado-lobuladas $1 / 2$ o más de su longitud, pediculadas, el pedículo $1-5 \mathrm{~cm}$, la pínnula basal basiscópica, $5-22 \mathrm{~cm}$, lobulada; raquis y costas pajizos, pardo-amarillentos o pardos, diminutamente pilosos o glabros en ambas superficies; aréolas generalmente sin nérvulos libres incluidos; tejido laminar glabro en ambas superficies o finamente peloso en el envés, los márgenes esparcidamente ciliados, especialmente en los senos; indusio reniforme a circular, unido lateralmente; esporas irregulares.

Etimología. Este híbrido es dedicado a Rafael Chacón, quien por mucho tiempo ha contribuido con la colección del Herbario Nacional de Costa Rica.

DistRIBUCION. Hasta ahora sólo se ha recolectado junto al Río Sarapiquí, en las cercanías de la Estación Biológica La Selva.

Paratipo. Costa Rica. Heredia: Sarapiquí, Puerto Viejo, Estación Biológica La Selva, orillas del Río Sarapiquí, $10^{\circ} 26^{\prime} 20^{\prime} \mathrm{N}, 8^{\circ} 00^{\prime} 40^{\prime} \mathrm{W}, 40-60 \mathrm{~m}, 25$ febr 1998, A. Rojas, E. Watkins y A. Soto 4369 (INB, CR, K, MO).

Este híbrido se diferencia de Tectaria acutiloba (Hieron.) Maxon por las escamas del rizoma más pequeñas [1.5-3 mm vs. (3-) 5-10 mm], pardo oscuro
( $v s$. pardo pálidas) y con superficie glabra ( $v s$. pubescente), frondas más grandes (1-1.4 $\mathrm{m} v s .0 .5-1$ $\mathrm{m}$ ), pinnas menos divididas (pinnado-lobuladas $v s$. pinnado-pinnatífidas) y yemas prolíferas en las axilas de las pinnas basales (vs. sin yemas) (Cuadro 1, Fig. 1 y 2). Difiere de T. lizarzaburui (Sodiro) C. Chr. por la lámina lanceolada (vs. lanceolado-oblonga), escamas del estípite de 2-5 mm de largo [vs. 1-3 $(-5) \mathrm{mm}$ ] y pardo oscuro (vs. negras), 0-1 (-2) pares de pinnas basales pinnado-lobuladas ( $v s$. todas las pinnas enteras o lobuladas) y lanceoladas ( $v s$. oblongas) y ápice de la lámina pinnatífido y gradualmente reducido ( $v s$. conforme o casi así y abruptamente reducido) (Cuadro 1, Figs. 1 y 2).

Para aclarar el concepto de T. andina (Baker) C. Chr., Rojas (2001) menciona el uso del nombre $T$. lizarzaburui (Sodiro) C. Chr. en lugar de T. vivipara Jermy \& T.G. Walker, pero sin anotar diferencias entre estas especies ni la distribución geográfica. Por tal motivo, aquí se aclaran esos puntos. En fotografías de tipos (NY, UC) se puede notar que $T$. lizarzaburui tiene frondas pinnadas con margen lobulado, (3-) 5-8 pares de pinnas, y las pinnas basales y medias presentan generalmente lóbulos basales, a veces en ambos lados. Contrariamente, para la mayoría de pteridólogos $T$. lizarzaburui es una planta con frondas pinnado-pinnatífidas (a veces hasta bipinnado-pinnatífidas), con 2-4 pares de pinnas; además, las escamas del rizoma son pardas, de (2-) 3-5 $\times$ 2-3 mm, en tanto que en el material continental de la supuesta $T$. vivipara las escamas del rizoma son negras, de 0.8-1.5 (-2) x 0.5-1 mm. El concepto de T. lizarzaburui según Smith (1995) y Tryon \& Stolze (1991) probablemente corresponde a una especie no descrita, más afín a T. transiens (C.V. Morton) A.R. Sm., tal como lo indica Smith (1995), dado que las escamas del rizoma son muy similares. No obstante, el material de México y Centroamérica presenta frondas pinnado-lobuladas, con pinnas oblongas y las incisiones no superan $1 / 4$ entre el margen y la costa, mientras que en el material sudamericano las frondas son comúnmente pinnadopinnatífidas, con pinnas lanceoladas a deltado-lanceoladas y las incisiones llegan hasta $1 / 2$ entre el margen y la costa.

En resumen, $T$. lizarzaburui difiere de $T$. vivipara 

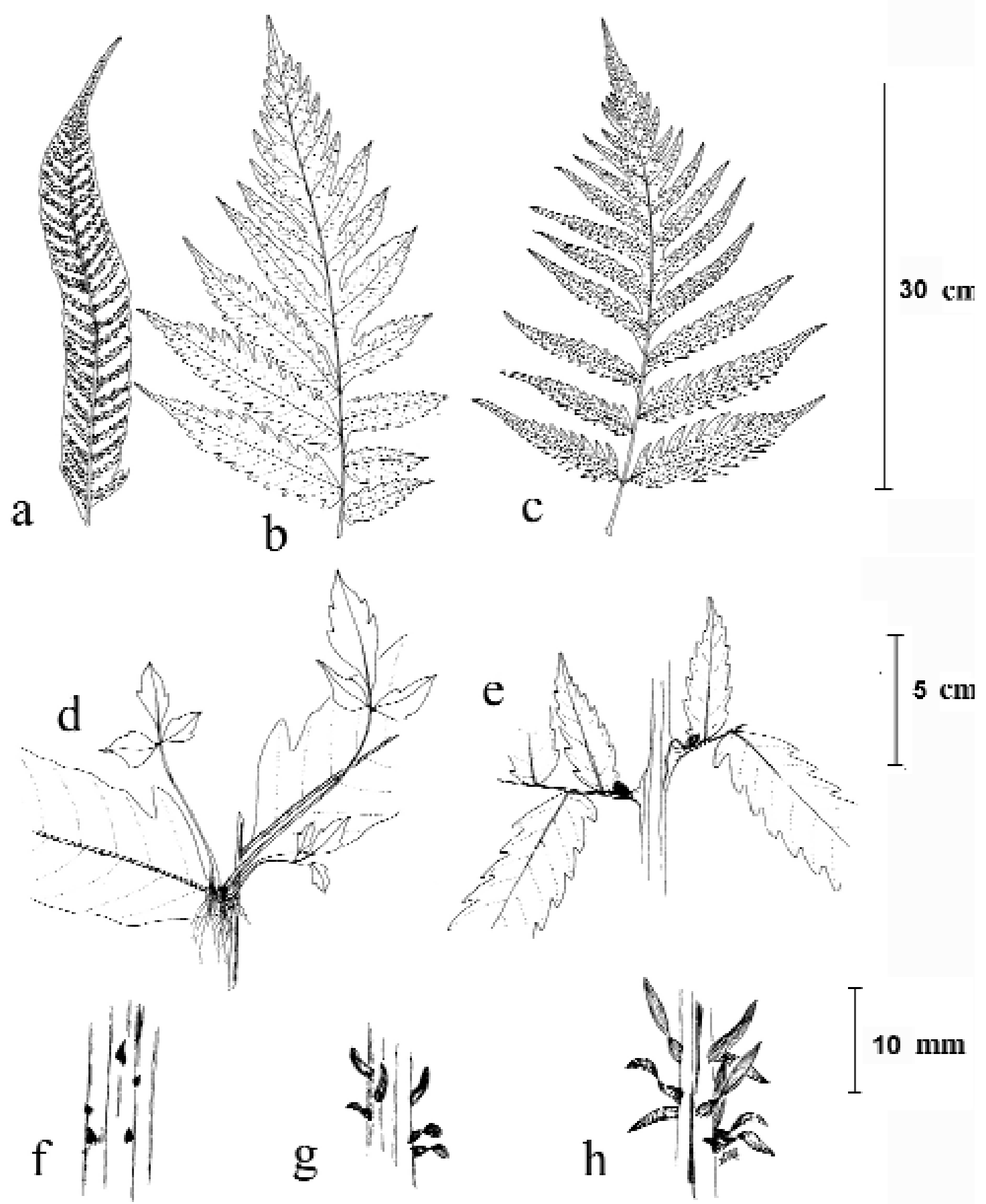

Fig. 1. Frondas de: a) Tectaria lizarzaburui (Sodiro) C.Chr. (A. Rojas et al. 4146, INB). b) Tectaria $\mathrm{x}$ chaconiana A. Rojas (A. Rojas et al. 4156, INB). c) Tectaria acutiloba (Hieron.) Maxon (A. Rojas et al. 4147, INB). 


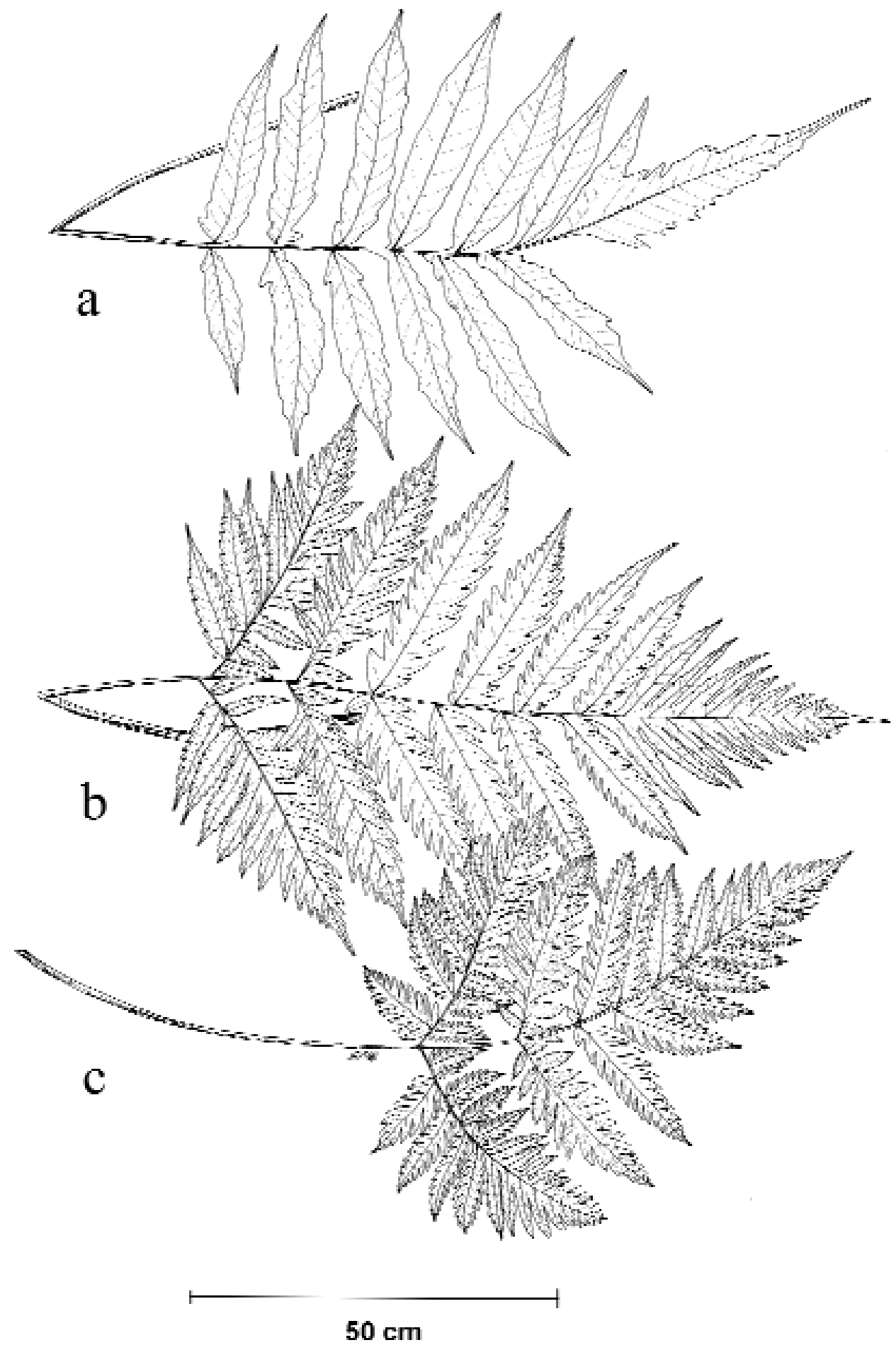

Fig. 2. Tectaria lizarzaburui (Sodiro) C.Chr. (A. Rojas et al. 4146, INB): a) Pinna. d) Base de las pinnas basales. f) Base del estípite. Tectaria x chaconiana A. Rojas (A. Rojas et al. 4156, INB): b) Pinna. e) Base de las pinnas basales. g) Base del estípite. Tectaria acutiloba (Hieron.) Maxon (A. Rojas et al. 4147, INB): c) Pinna. h) Base del estípite. 
Cuadro 1. Diferencias morfológicas entre Tectaria acutiloba, T. x chaconiana y T. lizarzaburui.

\begin{tabular}{|c|c|c|c|}
\hline Características & T. acutiloba & T. $x$ chaconiana & T. lizarzaburui \\
\hline Longitud de la fronda & $0.5-1 \mathrm{~m}$ & $1-1.4 \mathrm{~m}$ & $0.7-1.2(-1.6) \mathrm{m}$ \\
\hline Forma de la lámina & deltada & lanceolada & lanceolado-oblonga \\
\hline Escamas del rizoma & (3-) $5-10 \mathrm{~mm}$ & $1.5-3 \mathrm{~mm}$ & $1-3 \mathrm{~mm}$ \\
\hline Escamas del estípite & (3-) $5-10 \mathrm{~mm}$ & $2-5 \mathrm{~mm}$ & $1-3(-5) \mathrm{mm}$ \\
\hline Color de las escamas & pardo pálido & pardo oscuro & negro \\
\hline Superficie de las escamas & pubescente & glabra & glabra \\
\hline Pares de pinnas pinnado-incisas & $1-3$ & $0-1(-2)$ & 0 \\
\hline Pares de pinnas & $4-6$ & $5-7$ & $5-8$ \\
\hline Pinnas basales & oblongas & lanceoladas & lanceoladas \\
\hline Incisión de las pinnas & $\begin{array}{l}\text { pinnado- } \\
\text { pinnatífidas }\end{array}$ & $\begin{array}{c}\text { pinnatífidas a } \\
\text { pinnado-lobuladas }\end{array}$ & $\begin{array}{l}\text { enteras a } \\
\text { lobuladas }\end{array}$ \\
\hline Ápice de la lámina & gradualmente reducido & + gradualmente reducido & abruptamente reducido \\
\hline Yemas prolíferas & ausentes & presentes & presentes \\
\hline Esporas & simétricas & irregulares & simétricas \\
\hline
\end{tabular}

por las frondas más largas [70-130 cm de largo vs. 35-70 (-105) cm], el pecíolo pardo a atropurpúreo (vs. estramíneo a pardo-rojizo), la lámina más grande $(35-75 \times 30-50 \mathrm{~cm} v s .40-60 \times 25-32 \mathrm{~cm})$, las pinnas basales más grandes $(17-28 \times 3.5-4.5 \mathrm{~cm} v s$. $13-21 \times 2-3 \mathrm{~cm})$ y los soros en dos hileras entre las venas secundarias (vs. una serie). En cuanto a distribución geográfica también difieren, porque $T$. lizarzaburui habita en tierras continentales y $T$. vivipara está restringida a las islas del Caribe.

Tampoco Tectaria mexicana (Fée) C.V. Morton parece ser un caso sencillo, como lo indica Moran (Moran \& Riba 1995). Existen plantas con escamas del rizoma pelosas y otras con escamas glabras. También es variable la vellosidad de la lámina. Agregado a esto, pude notar que el material de la Isla del Coco es un tanto diferente por tener escamas del rizoma glabras ( $v s$. pelosas), pelos de la lámina 0.5-1 mm (vs. 0.1-0.5 mm) y por crecer en acantilados rocosos y húmedos, generalmente en escorrentías o cascadas ( $v s$. terrestres y a orillas de quebradas con pendiente inferior a $45^{\circ}$ ). Por otro lado, la entidad más diferente parece ser $T$. acutiloba
(Hieron.) Maxon, cuyo nombre ha sido considerado sinónimo de $T$. mexicana. Los caracteres diferenciales más importantes de T. acutiloba son los segmentos largos y agudos ( $v s$. cortos y obtusos), el tejido laminar glabro en ambas superficies o con pelos en el margen o cerca de él en el haz ( $v s$. peloso en el haz y peloso o glabro en el envés), el hábitat en márgenes de ríos, en suelos de sedimentación y en zonas con muy buena iluminación (en tanto T. mexicana habita a orillas de quebradas o en sotobosque, en suelos estables, más o menos ricos en materia orgánica, o sobre rocas y con poca iluminación), y la distribución a elevaciones entre 0 y $800 \mathrm{~m}[v s$. (100-) 600 y $2000 \mathrm{~m}]$.

Sin embargo, aún quedan por aclarar las variaciones en vellosidad que exhibe $T$. acutiloba, ya que en el material sudamericano el pecíolo y el raquis son glabros, en la vertiente Caribe desde Costa Rica hasta Belice los ejes son pelosos y las escamas del rizoma amarillentas y más largas; en las plantas de la Isla del Coco el haz de la lámina es peloso como en $T$. mexicana, pero los pelos son más dispersos y largos; además, los segmentos son agudos como en 
T. acutiloba y las escamas del rizoma son casi glabras. En la vertiente pacífica de Centroamérica hay plantas con el envés de la lámina cubierto de pelos glandulares. En resumen, es un grupo que requiere de más estudio. Tectaria acutiloba se extiende desde Honduras, en la vertiente atlántica, hasta Costa Rica, las zonas bajas de Panamá, pasando a la vertiente pacífica de Colombia y Ecuador, entre 0 y $600(-800) \mathrm{m}$, mientras que $T$. mexicana se distribuye en las cordilleras desde México hasta Ecuador, entre 600 y $1800(-2000) \mathrm{m}$.

Agradecimientos. Al Museo Nacional de Costa Rica por permitirme utilizar mi tiempo laboral en la corrección del artículo, a los herbarios consultados por permitirme utilizar sus colecciones, a los revisores anónimos del manuscrito por sus comentarios y a Francisco Quesada por sus excelentes ilustraciones.

\section{LITERATURA CITADA}

Gómez, L.D. 1977. Contribuciones a la Pteridología Centroamericana. IX. Novitates. Brenesia 10-11: 115119.

Grayum, M.H. 1987. On three misunderstood neotropical species of Tectaria (Polypodiaceae: Asplenioideae). Phytologia 64: 30-35.

Jermy, A. C. \& T.G. Walker. 1985. Cytotaxonomic studies of the ferns of Trinidad: 3. Descriptions of new species and hybrids and a new combination. Bull. Brit. Mus. (Nat. Hist.), Bot. 13(2): 251-256.
Mickel, J.T. \& J.M. Beitel. 1988. Pteridophyte Flora of Oaxaca, Mexico. Mem. New York Bot. Gard. 46: 357361.

Moran, R.C. 1990. Three new species of ferns from Mesoamerica. Ann. Missouri Bot. Gard. 77: 591-593. 1990.

Moran, R.C. 1992. Five new species of ferns from the American tropics. Novon 2: 137-138.

Moran, R.C. 1995. Tectaria. In: R.C. Moran \& R. Riba (eds.). Flora Mesoamericana. Vol. 1. Psilotaceae a Salviniaceae. Universidad Nacional Autónoma de México. México. p. 204-209.

Murillo, M.T. \& M.A. Harper. 1990. Helechos y plantas afines de Colombia. Rev. Acad. Col. Cie. Ex., Fís. Nat. Bogotá, Colombia. p. 229-233.

Rojas, A.F. 2001. Ocho nuevas especies y nuevos ámbitos geográficos de helechos de la familia Tectariaceae (Filicales) en el neotrópico. Rev. Biol. Trop. 49(2): 467-488.

Smith, A.R. 1981. Tectaria, Pteridophytes. In: D. Breedlove (ed.). Flora of Chiapas 2. California Acad. Sci., San Francisco. p. 214-216.

Smith, A.R. 1995. Tectaria. In: P. Berry, B. Holst \& K. Yatskievych (eds.). Flora of the Venezuelan Guayana. Vol. 2. Timber Press, Portland. p. 123-126.

Stolze, R.G. 1981. Ferns and fern allies of Guatemala. Part II. Polypodiaceae. Fieldiana, Bot., n.s. 6: 465-472.

Tryon, R.M. \& R.G. Stolze. 1991. Pteridophyta of Peru. Part IV. 17. Dryopteridaceae. Fieldiana, Bot., n.s. 27: 2-29.

Wagner, W.H., F.S. Wagner \& L.D. Gómez. 1978. The singular origin of a Central American fern, Pleuroderris michleriana. Biotropica 10: 254-264. 